\title{
Two-stage code reference beamformer for the reception of frequency hopping modulated signals ${ }^{\text {is }}$
}

\author{
Montse Nájar*, Xavier Mestre, Miguel A. Lagunas \\ Department of Signal Theory and Communications, Universitat Politècnica de Catalunya, c/. Jordi Girona, 1-3, 08034 Barcelona, Spain
}

Received 24 June 1999; received in revised form 15 May 2000

\begin{abstract}
This paper presents a beamforming technique for the reception of frequency hopping $(\mathrm{FH})$ modulated signals, which takes advantage of their inherent frequency diversity. This technique, based on a code reference beamformer, requires neither temporal nor spatial a priori reference and allows continuous self-calibration of the array. The proposed framework is composed of two different stages. The first stage employs the inverse of the noise plus interference covariance matrix obtained by an anticipative processor. The second stage makes use of the steering vector of the signal of interest which is adaptively obtained by maximizing the output signal to interference plus noise ratio (SINR). Using this information, the first stage is in turn readjusted and, as a result, the scheme is able to track non-stationary scenarios following the channel variations with no previous references other than knowledge of the frequency hopping sequence. The two-stage code reference beamformer provides the convergence rate necessary to avoid the SINR reduction associated with frequency hops in existing methods. (C) 2000 Elsevier Science B.V. All rights reserved.
\end{abstract}

\section{Zusammenfassung}

Dieser Artikel stellt ein Beamforming-Verfahren zum Empfang von Frequency-Hopping (FH) Signalen vor, welches ihre Frequenzdiversitätseigenschaften vorteilhaft ausnutzt. Diese Technik, die auf einem Code-Referenzbeamformer basiert, benötigt weder zeitliche noch räumliche a priori Kenntnisse und erlaubt eine fortlaufende selbst-Kalibration der Sensorgruppe. Das vorgeschlagene Verfahren besteht aus zwei unterschiedlichen Abschnitten. Der erste Schritt verwendet die Inverse der Rausch- plus Störsignal Kovarianzmatrix, die im Voraus geschätzt wird. Der zweite Schritt benutzt den zum interessierenden Signal gehörenden Richtungsvektor, der adaptiv durch Maximierung des Ausgangssignal- zu Stör- plus Rauschsignal- Verhältnisses (SINR) gewonnen wird. Auf Basis dieser Information wird der erste Schritt erneut durchlaufen, wodurch das Verfahren in der Lage ist, nichtstationären Bedingungen zu folgen, indem es sich an Kanaländerungen anpasst, ohne zurückliegende Referenzen zu benutzen sondern dabei nur die Kenntnis der Frequency Hopping Sequenz ausnutzt. Der Zweischritt- Code-Referenzbeamformer besitzt eine ausreichende Konvergenzrate, um die SINR-Reduktion, die mit existierenden Verfahren verbunden ist, zu vermeiden. (C) 2000 Elsevier Science B.V. All rights reserved.

\section{Résumé}

Cet article présente une technique de formation de voies pour la réception de signaux modulés par saut de fréquence, qui tire avantage de leur diversité de fréquences inhérente. Cette technique, basée sur un formateur de voies par référence

\footnotetext{
${ }^{2}$ Partially supported by the Spanish Government (CICYT) TIC98-0412, TIC98-0703, (CIRIT) 1998 SGR 00081.

* Corresponding author. Tel.: 34-3-4017051; fax: 34-3-4016447.

E-mail address: najar@gps.tsc.upc.es (M. Nájar).
} 
de code, ne nécessite ni référence temporelle ni spatiale a priori, et permet une auto-calibration continue du réseau. Le cadre de travail proposé est composé de deux étapes différentes. La premiére étape utilise l'inverse de la matrice de covariance du bruit plus de l'interférence obtenue par un processeur anticipatif. La seconde étape utilise le vecteur d'orientation du signal d'intérét qui est obtenu de façon adaptative en maximisant le rapport signal à interférence plus bruit (SINR). En utilisant cette information, la première étape est réajustée à son tour et, comme résultat, le schéma est capable de suivre des scénarios non stationnaires en suivant les variations du canal sans références préalables autres que la connaissance de la séquence de saut fréquentiel. Le formateur de voies par référence de code fournit le taux de convergence nécessaire pour éviter une réduction du SINR associée aux sauts de fréquence dans les méthodes existantes. (C) 2000 Elsevier Science B.V. All rights reserved.

Keywords: Code reference beamforming; Frequency hopping modulation; Mobile communication

\section{Introduction}

The application of frequency hopping $(\mathrm{FH})$ modulation to array processing results in a great capacity for interference rejection due to the combination of frequency diversity, in the modulation, and spatial diversity, at the array. Existing beamformers for the reception of $\mathrm{FH}$ modulated signals are based on classical techniques of temporal or spatial reference beam-forming. First, Acar and Compton [1] proposed the application of the least mean square (LMS) algorithm in a time reference adaptive array. Next, Bakhru and Torrieri [2] presented the Maximin algorithm, a specific method for adaptive arrays using FH signals, based on the spectral characteristics of these signals. Finally, Eken [4] proposed a modified sidelobe canceller for $\mathrm{FH}$ signals that needs a priori knowledge of the direction of arrival of the desired signal. The main limitation of the previous approaches is the fall of SINR at the hop instants. The reason for this behavior is that changes in signal frequency due to $\mathrm{FH}$ modulation are seen by the algorithm as changes in the direction of arrival of co-channel interference, resulting in discontinuities of the adaptive algorithm's performance.

This paper deals with a new beamforming technique especially appropriate for $\mathrm{FH}$ modulated signals. The approach takes advantage of the knowledge of the frequency hopping sequence at the receiver and requires neither a reference signal nor a spatial a priori reference. The basic principle used in the proposed system is the existing differences between the array covariance matrices obtained in two consecutive hops. Several authors in
non-FH contexts have already used this property. First, Viberg [12] considered the signal of interest inactive during some time intervals. Therefore, the covariance matrix due only to the interference and noise contributions can be estimated during these intervals. Later, Souloumiac [10] proposed to obtain the optimum beamforming weight vector from two covariance matrices estimated in different time intervals, being the desired signal power distinct between both intervals. Another beamforming method based on the estimate of two different covariance matrices is proposed by Suard [11]. This reference deals with a cellular mobile communication system with code division multiple access and synchronous direct sequence modulation (DS-CDMA). The two covariance matrices used are obtained before and after the correlation of the received signal vector with the spreading code.

The approach presented in this paper, named code reference beamformer, is implemented in two separated stages in order to avoid the drop in SINR associated with frequency hops. The first stage embodies the inverse of the noise plus interference covariance matrix, which can be estimated by an anticipative processor operating at the frequency of the next hop. The second stage is a beamforming weight vector equal to the steering vector of the desired signal, adaptively obtained by maximizing the SINR at the array output. The estimate will be derived by means of a gradient algorithm that presents a higher convergence rate than previously described methods [7] thanks to the first preprocessing stage.

The paper is organized as follows: First, the data model is presented in Section 2. Next, in Section 3, 
the two-stage code reference beamformer is introduced and its application to mobile communications scenarios is proposed. Results from computer simulations are exposed in Section 4. Finally, Section 5 offers some concluding remarks.

\section{Data model}

The input signal of an array of $Q$ isotropic sensors placed at arbitrary positions illuminated by $M$ impinging plane waves and narrow band signals can be modeled as:

$\boldsymbol{x}(t)=\sum_{s=1}^{M} a_{s}(t) \boldsymbol{s}\left(\boldsymbol{k}_{s i}\right)+\boldsymbol{n}(t)$,

where $\boldsymbol{x}(t)$ and $\boldsymbol{n}(t)$ are the snapshot and noise $Q$-dimensional vectors, respectively; $a_{s}(t)$ is the analytic signal corresponding to the sth source and $\boldsymbol{s}\left(\boldsymbol{k}_{s i}\right)$ is its steering vector, which can be represented in (2), being $g_{q i}$ the gain of the $q$ th element at the $i$ th frequency and $\psi_{q s i}=-j \boldsymbol{k}_{s i} \boldsymbol{x}_{q}+\varphi_{q i}$ the phase introduced at the $q$ th sensor, which depends on its location $\boldsymbol{x}_{q}=\left\lfloor x_{q}, y_{q}, z_{q}\right\rfloor^{\mathrm{T}}$, the wave vector $\boldsymbol{k}_{s i}=2 \pi / \lambda_{i}\left[\sin \theta_{s} \cos \phi_{s}, \sin \theta_{s} \sin \phi_{s}, \cos \theta_{s}\right]$ and the uncompensated phase error $\varphi_{q i}$. The wave vector depends in turn on the signal wavelength corresponding to the $i$ th frequency $\lambda_{i}$, and on the $s$ th signal direction of arrival, determined by its elevation and azimuth angles, $\theta_{s}$ and $\phi_{s}$, respectively,

$\boldsymbol{s}\left(\boldsymbol{k}_{\boldsymbol{s} i}\right)=\left[g_{1 i} \mathrm{e}^{\psi_{1 s i}}, g_{2 i} \mathrm{e}^{\psi_{2 s i}}, \ldots, g_{Q i} \mathrm{e}^{\psi_{Q s i}}\right]^{\mathrm{T}}$.

The signal at the array output is equal to the combination of the signals received at the sensors, properly weighted. It is well known that the weight vector that maximizes the output SINR is proportional to the product of the inverse of the noise plus interference covariance matrix $\boldsymbol{R}_{n}^{-1}$ and the steering vector of the signal of interest, one of the vectors $\boldsymbol{s}\left(\boldsymbol{k}_{s i}\right)$, denoted thereafter as $\boldsymbol{s}_{\mathrm{d}}$ :

$\boldsymbol{w}_{\mathrm{opt}}=k \boldsymbol{R}_{n}^{-1} \boldsymbol{s}_{\mathrm{d}}$.

In mobile communications systems, due to multipath propagation, several replicas of the transmitted signals are received from different directions and with distinct time delays. This phenomenon, along with the presence of doppler, will cause the characteristic fading effects on the received signals. In this case, the solution that maximizes the SINR at the array output is still (3), although now $\boldsymbol{s}_{\mathrm{d}}$ stands for a generalized steering vector that depends on time due to channel fading:

$\boldsymbol{s}_{\mathrm{d}}(t)=\frac{1}{N} \sum_{i=1}^{N} \alpha_{i}(t) \boldsymbol{s}_{\mathrm{d} i}$

with $N$ the number of propagation paths, $\alpha_{i}(t)$ the reflection coefficient for path $i$, and $\boldsymbol{s}_{\mathrm{d} i}$ the steering vector associated with the corresponding moving source.

\section{Two-stage code reference beamformer}

The aforementioned two processing stages become the crucial advantage of the code reference available in FH systems, allowing optimum beamforming without a priori information about the scenario. The proposed framework is composed of two parallel processors (Fig. 1).

The aim of the anticipative processor is to estimate the matrix $\boldsymbol{R}_{n}^{-1}$ required in the first stage of the beamformer beforehand from the snapshots acquired at the anticipative dehopping output. The

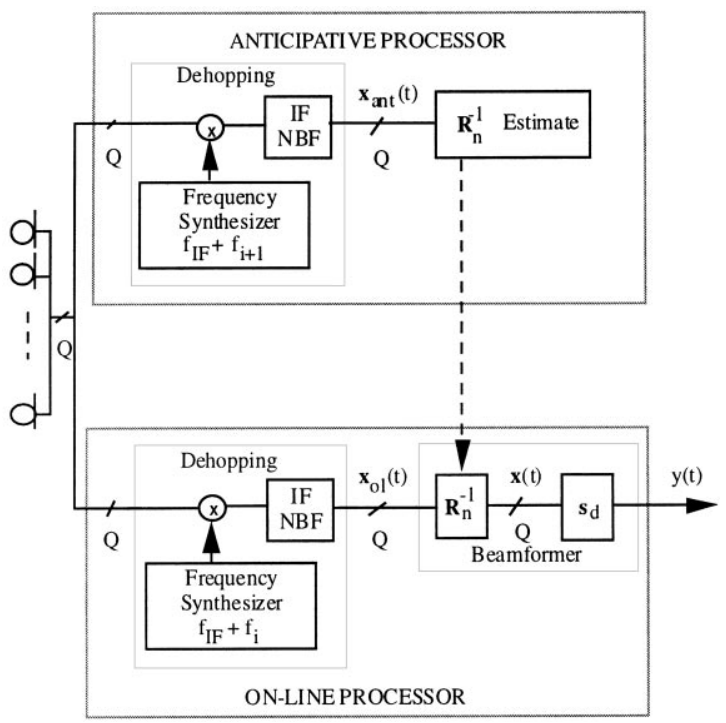

Fig. 1. Code Reference Beamformer. 


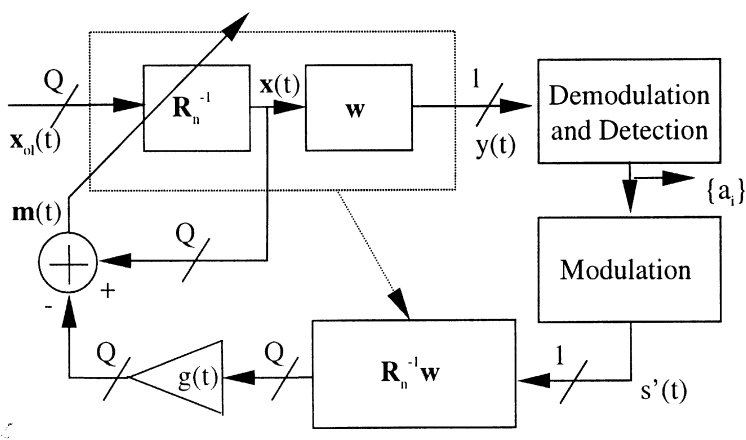

Fig. 2. First stage adaptation for frequency-non-selective channels.

resulting snapshot $\boldsymbol{x}_{\text {ant }}(t)$ contains the noise and the interference that will appear in the scenario after the next hop, assuming that the interfering sources stay spatially stationary along the time interval between two consecutive jumps. In mobile radio communication scenarios, due to the presence of fading on the received interference, the first stage estimated by the anticipative processor is adequate only as an initialization of the system [6]. Once it has been transferred to the on-line processor and after few iterations aimed to achieve the second stage convergence, the first stage is continuously updated (Fig. 2) using the following recursion:

$\boldsymbol{R}_{n}^{-1}(i+1)=\gamma \boldsymbol{R}_{n}^{-1}(i)+(1-\gamma) \boldsymbol{m}(i+1) \boldsymbol{m}^{\mathrm{H}}(i+1)$

with $\gamma$ a forgetting factor and $\boldsymbol{m}(i)$ an estimation of the undesired component of the incoming snapshot preprocessed by the first stage of the beamformer:

$\boldsymbol{m}(i)=\boldsymbol{x}(i)-g(i) s^{\prime}(i) \boldsymbol{R}_{n}^{-1} \boldsymbol{w}$

where $s^{\prime}(i)$ is a noiseless regenerated signal that can be extracted from the beamformer output $y(i)$ up to a phase ambiguity depending on the modulation format and $g(i)$ is an adaptive coefficient continuously adjusted to minimize the log-likelihood cost function $\boldsymbol{m}^{\mathrm{H}}(i) \boldsymbol{R}_{n}^{-1} \boldsymbol{m}(i)$.

In practice, it will be obviously impossible for the coefficient $g(i)$ to completely suppress the desired signal component in $x(i)$. This results in a residual presence of desired signal in the estimated matrix used as first stage of the beamformer. This effect is equivalent to the lack of synchronism at the dehop- ping stage. In [7], it is proved that the convergence rate with leakage of the desired signal into the covariance matrix can be maintained with an increment in the algorithm misadjustment.

The on-line processor maximizes the SINR at the array output. The first stage multiplies the snapshot $\boldsymbol{x}_{\mathrm{o} 1}(t)$ by the inverse of the interference plus noise correlation matrix $\boldsymbol{R}_{n}^{-1}$, which is previously estimated at the anticipative processor and subsequently transferred to this processor right after the frequency hop occurs. The second stage is devoted to focus the beampattern on the signal of interest, weighting the preprocessed signal vector $\boldsymbol{x}(t)$ with the desired steering vector $\boldsymbol{s}_{\mathrm{d}}$. In general, this beamforming weight vector is unavailable because the knowledge of the direction of arrival of the signal of interest would be hardly precise enough to be used without pointing errors. Furthermore, the steering vector may contain numerous calibration errors such as sensor placement errors, phase and magnitude perturbations in the sensor gains or even coupling effects among array elements. This drawback, common in all spatial reference beamformers, is overcome by the twostage code reference beamformer, in which the steering vector is adaptively obtained by maximizing the output SINR.

$\mathrm{SINR}=\frac{\boldsymbol{w}^{\mathrm{H}} \boldsymbol{R}_{x} \boldsymbol{w}}{\boldsymbol{w}^{\mathrm{H}} \boldsymbol{R}_{n}^{-1} \boldsymbol{w}}-1$,

where $\boldsymbol{R}_{x}$ is the correlation matrix at the output of the first stage and $\boldsymbol{w}$ is the weight vector to be estimated. The inverse of the noise plus interference correlation matrix $\boldsymbol{R}_{n}^{-1}$, used to preprocess the incoming snapshots, yields the correlation matrix of the undesired signals at the first stage output.

The SINR maximization can be interpreted as a constrained maximization of the array output power:

$\max _{w} \boldsymbol{w}^{\mathrm{H}} \boldsymbol{R}_{x} \boldsymbol{w} \quad$ subject to $\quad \boldsymbol{w}^{\mathrm{H}} \boldsymbol{R}_{n}^{-1} \boldsymbol{w}=1$.

The solution to this problem can be found to be the generalized eigenvector of the pencil $\left(\boldsymbol{R}_{x}, \boldsymbol{R}_{n}^{-1}\right)$ corresponding to the maximum generalized eigenvalue $\lambda_{\max }$ equal to the array output power:

$\boldsymbol{R}_{x} \boldsymbol{w}_{\mathrm{opt}}=\lambda_{\max } \boldsymbol{R}_{n}^{-1} \boldsymbol{w}_{\mathrm{opt}}$. 
The adaptation rule of the instantaneous gradient algorithm we suggest is the following:

$$
\begin{aligned}
\boldsymbol{w}(i & +1) \\
& =\boldsymbol{w}(i)+\frac{\alpha}{\delta_{\max }(\lambda(i)-1)}\left(\boldsymbol{x}(i) y(i)^{*}-\lambda(i) \boldsymbol{R}_{n}^{-1} \boldsymbol{w}(i)\right),
\end{aligned}
$$

where the adaptation parameter has been normalized, so the trade-off between convergence rate and misadjustment will uniquely depend upon the constant $\alpha$. The constant $\delta_{\max }$ represents the inverse of the noise power and $\lambda(i)$ denotes the maximum generalized eigenvalue, which corresponds to the estimated power at the array output:

$\lambda(i)=\beta \lambda(i-1)+(1-\beta)|y(i)|^{2}$.

The Appendix proves that this procedure provides the generalized eigenvector corresponding to the maximum generalized eigenvalue of the pencil $\left(\boldsymbol{R}_{x}, \boldsymbol{R}_{n}^{-1}\right)$. This eigenvector yields the desired steering vector $\boldsymbol{s}_{\mathrm{d}}$, and, thereby the optimum weight vector (3) is obtained.

An alternative mechanism to speed up convergence rate is frequency focusing, a technique which allows acceleration of the convergence rate for the presented adaptive algorithm taking advantage of the information obtained before a particular frequency hop. Furthermore, this technique succeeds in reducing SINR falls at the frequency hop instants. The convergence of the beamforming weight vector estimate to its optimum value in a particular hop yields the steering vector of the signal of interest $\boldsymbol{s}_{\mathrm{d}}$ which can be generically expressed by Eq. (2). Hence, assuming that the phases of the array sensors are calibrated, the steering phases can be disposed as proportional to the current frequency (module $2 \pi$ ). Consequently, frequency focusing permits the prediction of the optimum beamforming weight vector for the next hop. This procedure does not require previous knowledge either of the location of sensors or of the desired direction of arrival. However, it is generally accepted that in frequency jumping systems the frequency spacing is always set higher than the coherence bandwidth of the mobile channel. Therefore, two consecutive channels will appear as statistically independent, and the same will happen to the generalized steering vectors $\boldsymbol{s}_{\mathrm{d}}(t)$.
This fact, along with the lack of linear dependence on the frequency of the equivalent steering vector elements, might contribute to a degradation of the results achieved with the frequency focusing process. Nevertheless, simulation results show that it is still adequate to focus the second stage at the hop instants, since there is always spatial information that can be taken into account in order to obtain a proper initialization of the weight vector for next frequency hop.

The code reference beamformer presented in this paper can be extended to the case of frequencyselective channels. As it is shown in [9], a slowly time-varying frequency-selective channel can be modeled as a tapped delay line with time varying weights, being the tap spacing approximately equal to the inverse of the signal bandwidth. Thereby, the signature of a particular user on the array can be expressed as a linear combination of responses of delayed versions of the emitted signal to a set of frequency-non-selective equivalent channels:

$\boldsymbol{x}_{u}(t)=\sum_{i} a_{u}(t-i T) \boldsymbol{s}_{\mathrm{ui}}(t)$

with $a_{u}(t)$ denoting the source signal of the $u$ th user, $\boldsymbol{s}_{\mathrm{ui}}(t)$ the generalized steering vector corresponding to the $i$ th narrow band component of the channel and $T$ the time spacing between taps of the model. Note from (12) that, in contrast to the frequencynon-selective channel case, the signal correlation matrix associated with each user does not present dyadic structure and, as a consequence, classical beamforming techniques are no longer appropriate. The problem of designing specific beamforming techniques for scenarios with coherent sources has been thoroughly treated by Bresler et al. in [3].

In frequency-selective channels the continuous adaptation of the matrix calculated in the anticipative processor and transferred to the on-line processor, is more complicated than in the non-selective case. On the one hand, it is not possible to directly regenerate the desired signal at the array input from the detected data because it is necessary to include the multipath effect. Besides, some sort of blind equalizer is needed in order to yield unbiased estimates of the data, but this is a common problem in all blind-detection systems and will not be specifically addressed herein. On the other hand, the 


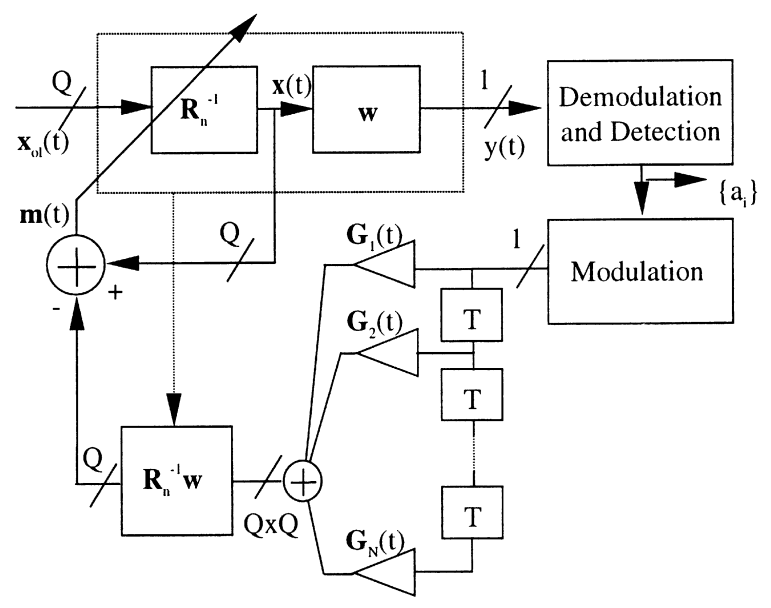

Fig. 3. First stage adaptation for frequency-selective channels.

second stage of the Code Reference Beamformer does not yield the generalized steering vector of the desired signal, although it converges to a linear combination of the different generalized steering vectors associated with different narrow band components of the channel. This stage is blindly estimated by an output SINR maximization process.

The system proposed to allow the adaptation of the first stage of the beamformer is represented in Fig. 3. Delayed versions of the regenerated signal are multiplied by a set of matrices of coefficients $\boldsymbol{G}_{i}$ devoted to the separation of distinct narrow band channel components associated with different resolvable times of arrival. Thanks to these matrices, the system is able to separate and thereafter properly combine the distinct narrow band channel contributions. Consequently, the suggested system is able to provide a reconstruction of the desired signal in the form that it is received by the array. This regenerated signal is finally intended to directly actualize the inverse of the noise plus interference covariance matrix, like in the frequency-nonselective channel case.

\section{Simulation results}

In this section we present some simulations to show the performance of the new two-stage Code Reference Beamforming proposed in this paper.
The simulations were made with a linear equally spaced array of 4 sensors, in which the interelement separation was half wavelength at the center frequency of the hopping band.

The first simulation was designed to evaluate the behavior of the array processing system proposed in Fig. 2 when dealing with frequency-non-selective mobile channels. The array was illuminated from elevations of 20 (desired user), -30 and $40^{\circ}$ (interfering users), being their signals uniformly spread over a $50 \%$ relative bandwidth. All the permitted frequencies of the system were simultaneously used by the interfering mobiles. Each user's flat-fading channel was generated with a ray model; particularly, a Laplacian power angular spectrum with a power angular spread (PAS) of $8^{\circ}$ was assumed for all channels and users. The direction of arrival of each user's rays was controlled by a Gaussian random variable with a dispersion of PAS/1.38 (experimental value), being its dynamic range truncated to the interval of elevations [ - 90,90] degrees. Naturally, both the central angle of the power angular spectrum and the mean ray elevation were set equal to the angular elevation of the corresponding user. In addition, the number of impinging wavefronts was generated by a Poisson random variable with mean equal to 25 rays. All mobile stations transmitted GMSK signals (4 samples/symbol) with a normalized $3 \mathrm{~dB}$ cut-off frequency of the Gaussian filter of 0.3 , and a mean received $\mathrm{Eb} / \mathrm{No}$ of $15 \mathrm{~dB}$. The speed of the users was fixed to $120 \mathrm{~km} / \mathrm{h}$, generating a classical Doppler Spectrum within $\pm 100 \mathrm{~Hz}$. The symbol period and the dwell time were adjusted to $3.6 \mu$ s and $0.54 \mathrm{~ms}$ (150 symbols), respectively, values intentionally chosen on account of their similarity to those proposed as symbol and slot length by the GSM standard. The simulation length was fixed to the duration of 1050 symbols, so 7 frequency hops occurred. Regarding the adaptation of the first stage, the coefficient $g(n)$ was updated using a LMS algorithm and the received signal was incoherently demodulated by means of a differential detector. The evolution of the instantaneous SINR with (solid line) and without adaptation of the first stage (dashdot line) has been depicted in Fig. 4, along with the optimum values (dotted line). It can be noted that the adaptation of the first stage confers 


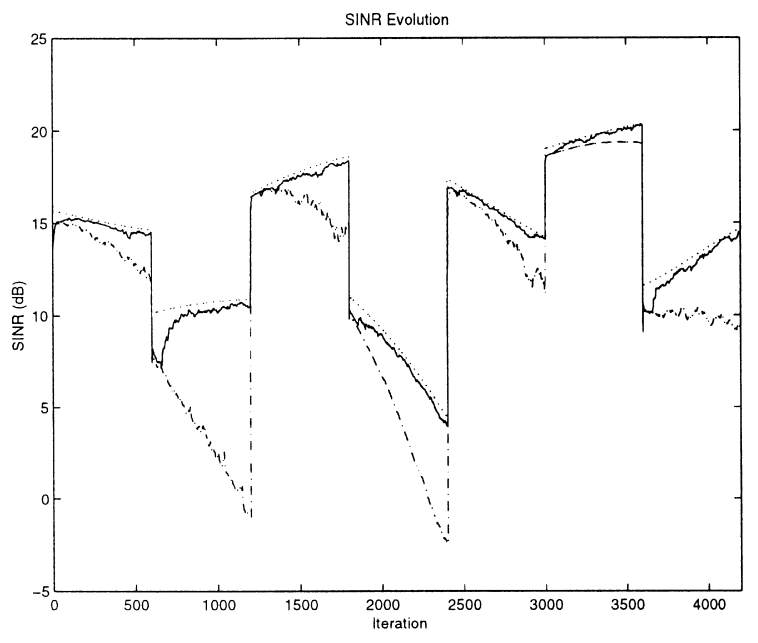

Fig. 4. Scenario constituted of three users transmitting GMSK signals $(\mathrm{BT}=0.3)$ from DOAs: $20^{\circ},-30^{\circ}$ and $40^{\circ}(\mathrm{Eb} / \mathrm{No}=$ $15 \mathrm{~dB}$ for all users) through a frequency-non-selective mobile channel $(v=120 \mathrm{~km} / \mathrm{h})$. Solid line: estimated beamforming with adaptation of the first stage. Dashdot line: estimated beamforming without adaptation of the first stage. Dotted line: optimum values.

the ability to track the channel variations with neither spatial nor temporal references apart from the knowledge of the frequency hopping sequence (knowledge that, remarkably, does not represent any overhead to the communication system). Besides, the plot clearly illustrates the reduction of the SINR falls at the hop instants, thanks not only to the previous estimation of the first stage, but also to the frequency focusing process.

We finally draw our attention upon the case in which signals are not only distorted by a non-stationary multiplicative channel, but also convolutedly affected by its frequency selectivity. A wideband channel was generated according to the signal model presented in (12) and following the GSM recommendations [5] with reference to the generation of frequency selectivity for urban areas (Typical Urban TUx). Specifically, the channel model referred as TU120 (speed $120 \mathrm{~km} / \mathrm{h}$ ) was simulated, where the frequency-flat channel model presented above was utilized to generate each narrow band multiplicative component of the global response. All other parameters were left as in previous simulations, even though now the system

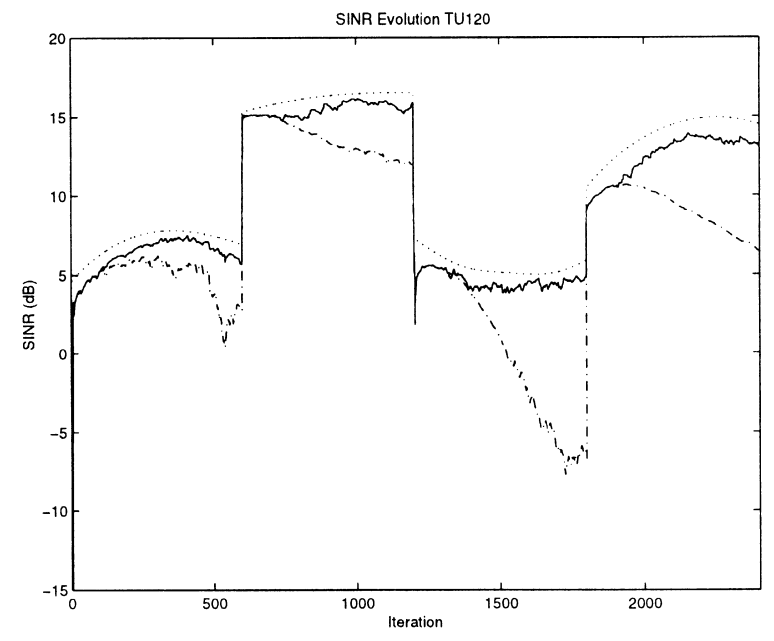

Fig. 5. Scenario constituted of three users transmitting MSK signals from DOAs: $20^{\circ},-30^{\circ}$ and $40^{\circ}(\mathrm{Eb} / \mathrm{No}=15 \mathrm{~dB}$ for all users) through a frequency-selective mobile channel: Urban area (TU120). Solid line: estimated beamforming with adaptation of the first stage. Dashdot line: estimated beamforming without adaptation of the first stage. Dotted line: optimum values.

under test was the one depicted in Fig. 3, where the time interval $T$ was set to $0.9 \mu$ s ( 1 sample). Hence, only six matrices $\boldsymbol{G}_{i}$ were necessary to process the maximum length of the channel. Again, each matrix was adjusted with an LMS-like algorithm in order to minimize the norm of the vector $\boldsymbol{m}(n)$. Fig. 5 shows the instantaneous SINR evolution with (solid line) and without adaptation of the first stage of the beamformer (dashdot line) along with the optimum values (dotted line). Clearly, these plots point out the high adaptability of the beamformer to non-stationary scenarios in presence of frequency-selective mobile channels.

\section{Conclusions}

A new beamformer for the reception of $\mathrm{FH}$ modulated signals has been presented in this paper. This new beamformer, based on code reference, is composed of two different stages. This particularity confers a high convergence rate and provides a considerable reduction of the SINR falls at the frequency hops. This reduction of the SINR falls is 
further increased by the application of frequency focusing of the second stage of the beamformer. From the signal preprocessed by the inverse of the noise plus interference covariance matrix, obtained in the anticipative processor, the second stage is adaptively estimated. This estimation consists of maximizing the SINR through a simple algorithm that requires no previous information about neither the array nor the scenario. In conclusion, continuous self-calibration of the array is attained. Furthermore, the system is able to follow the channel variations in a mobile communications environment, tracking non-stationary scenarios without need for any additional information.

\section{Appendix}

The objective of this appendix is to evaluate the convergence in the mean of the proposed algorithm. For this evaluation, the adaptive rule is expressed as an equation with an exponent

$\boldsymbol{w}(i+1)=\left[\boldsymbol{I}-\mu\left(\lambda_{\max } \boldsymbol{R}_{n}^{-1}-\boldsymbol{R}_{x}\right)\right]^{i+1} \boldsymbol{w}(0)$.

Expressing the difference matrix in terms of its eigenvalues and eigenvectors $\boldsymbol{M}=\lambda_{\max } \boldsymbol{R}_{n}^{-1}-$ $\boldsymbol{R}_{x}=\boldsymbol{Q} \Gamma \boldsymbol{Q}^{\mathrm{H}}$ and considering $\boldsymbol{Q} \boldsymbol{Q}^{\mathrm{H}}=1$, the above expression can be formulated as

$\boldsymbol{w}(i+1)=\boldsymbol{Q}(\boldsymbol{I}-\mu \boldsymbol{\Gamma})^{i+1} \boldsymbol{Q}^{\mathrm{H}} \boldsymbol{w}(0)$.

Therefore, convergence of the gradient algorithm defined in (10) will be determined by the elements of the diagonal matrix $\Gamma$ or, equivalently, by the eigenvalues of the difference matrix $\boldsymbol{M}$. One of these eigenvalues, the one associated with the main generalized eigenvector of the pencil $\left(\boldsymbol{R}_{x}, \boldsymbol{R}_{n}^{-1}\right)$, is zero; the rest of the eigenvalues are all positive. In consequence, the convergence of the beamforming weight vector estimate to its optimum value is assured by choosing a proper value for the adaptation constant

$0<\mu<\frac{2}{\gamma_{\max }}$.

Under this constraint, when $n$ tends to infinity, $(\boldsymbol{I}-\mu \boldsymbol{\Gamma})^{i+1}$ will tend to a matrix with only one element different from zero, the one corresponding to the null eigenvalue of $\boldsymbol{M}$, which is equal to one. Thereby, the estimated vector will converge to the optimum beamforming weight vector with a proportionality constant which depends on the algorithm initialization $\boldsymbol{w}(0)$

$\boldsymbol{w}(i+1)=\boldsymbol{w}_{\mathrm{opt}} \boldsymbol{w}_{\mathrm{opt}}^{\mathrm{H}} \boldsymbol{w}(0)=k \boldsymbol{w}_{\mathrm{opt}}$.

Hence, the minimum convergence rate required for this gradient algorithm is proportional to the eigenvalue spread of the matrix $\boldsymbol{M}$, defined as the ratio between the maximum and the minimum non-zero eigenvalue. Next, it is shown that this eigenvalue spread coincides with the eigenvalue spread of the inverse of the noise plus interference covariance matrix.

From the signal correlation matrix after the first stage preprocessing

$\boldsymbol{R}_{x}=\sigma_{\mathrm{d}}^{2} \boldsymbol{R}_{\mathrm{n}}^{-1} \boldsymbol{s}_{\mathrm{d}} \boldsymbol{s}_{\mathrm{d}}^{\mathrm{H}} \boldsymbol{R}_{n}^{-1}+\boldsymbol{R}_{n}^{-1}$,

the difference matrix $\boldsymbol{M}$ can be written as

$\boldsymbol{M}=\left(\lambda_{\max }-1\right) \boldsymbol{R}_{n}^{-1}-\sigma_{\mathrm{d}}^{2} \boldsymbol{R}_{n}^{-1} \boldsymbol{s}_{\mathrm{d}} \boldsymbol{s}_{\mathrm{d}}^{\mathrm{H}} \boldsymbol{R}_{n}^{-1}$.

Substituting, the inverse of the noise plus interference covariance matrix by its eigendecomposition $\boldsymbol{R}_{n}^{-1}=\boldsymbol{U} \Lambda \boldsymbol{U}^{\mathrm{H}}$ and defining the vector $\boldsymbol{z}=\boldsymbol{U}^{\mathrm{H}} \boldsymbol{R}_{n}^{-1} \boldsymbol{s}_{\mathrm{d}}$, it results in

$\boldsymbol{M}=\boldsymbol{U}\left(\left(\lambda_{\max }-1\right) \boldsymbol{\Lambda}-\sigma_{\mathrm{d}}^{2} \boldsymbol{z}^{\mathrm{H}}\right) \boldsymbol{U}^{\mathrm{H}}$.

The eigenvalue decomposition of matrix $\boldsymbol{M}$ can be obtained from the eigenvalues $\gamma$ and the eigenvectors $\boldsymbol{e}$ of the matrix in brackets of the above equation

$\left(\left(\lambda_{\max }-1\right) \boldsymbol{\Lambda}-\sigma_{\mathrm{d}}^{2} z \boldsymbol{z}^{\mathrm{H}}\right) \boldsymbol{e}=\gamma \boldsymbol{e}$.

The eigenvalues of both matrices are equal, whereas the eigenvectors of $\boldsymbol{M}$ can be derived from the product of the matrix $\boldsymbol{U}$ with each one of the eigenvectors $\boldsymbol{e}$. Therefore, the eigenvalues $\gamma$ can be obtained from the solutions of the next function [8]

$\frac{1}{\sigma_{\mathrm{d}}^{2}}=\sum_{q=1}^{Q} \frac{-\left|z_{q}\right|^{2}}{\gamma-\left(\lambda_{\max }-1\right) \delta_{q}}$,

where $\delta_{q}$ are the eigenvalues of the matrix $\boldsymbol{R}_{n}^{-1}$ and $z_{q}$ are the components of the vector $z$.

The resolution of this equation consists in the extraction of the zeros of a raising function. Each eigenvalue $\gamma_{q}$ is localized at an open interval 
\rfloor$\left(\lambda_{\max }-1\right) \delta_{q-1},\left(\lambda_{\max }-1\right) \delta_{q} L, \quad$ defined between two consecutive eigenvalues of the inverse of the noise plus interference covariance matrix. In consequence, the maximum eigenvalue of the matrix $\boldsymbol{M}$ is limited by the product of the maximum eigenvalue $\delta_{\max }$, equal to the inverse of the noise power assuming the noise is spatially white, and the maximum signal-to-noise plus interference ratio $\operatorname{SINR}_{\mathrm{d}}=\left(\lambda_{\max }-1\right)$ :

$\gamma_{\max } \leqslant \delta_{\max } \mathrm{SINR}_{\mathrm{d}}$.

The minimum eigenvalue of $\boldsymbol{M}$ is equal to zero, but the convergence rate of the adaptive algorithm is determined by the minimum eigenvalue different from zero, which is in turn limited by the minimum eigenvalue of the inverse of the noise plus interference covariance matrix, $\gamma_{\min \neq 0}=\left(\lambda_{\max }-1\right) \delta_{\min }$. Hence, the maximum eigenvalue spread of the matrix $\boldsymbol{M}$ coincides with the eigenvalue spread of the inverse of the noise plus interference covariance matrix:

$\chi(\boldsymbol{M})_{\max }=\chi\left(\boldsymbol{R}_{n}^{-1}\right)$.

Now, let us concentrate on the severe case of scenarios where interference is much stronger than the non-directional noise (INR $>10 \mathrm{~dB}$ ). If the level of the interfering signals is high, the matrix $\boldsymbol{R}_{n}^{-1}$ will present reduced eigenvalues. Particularly, if this level is much higher than the noise level, $\boldsymbol{R}_{n}^{-1}$ can be considered orthogonal to the interference space. Thus, this matrix will present eigenvalues close to zero yielding an unbounded eigenvalue spread of matrix $\boldsymbol{M}$; therefore, the convergence time of the gradient algorithm in this ill-conditioned case would tend to infinity. Considering the extreme case in which the eigenvalues of $\boldsymbol{M}$ corresponding to the interference space are null, the estimated beamforming weight vector for $n$ tending to infinity results equal to (A.12)

$\boldsymbol{w}(i)=\boldsymbol{Q}_{\mathrm{s}} \boldsymbol{Q}_{\mathrm{s}}^{\mathrm{H}} \boldsymbol{w}(0)$,

where $\boldsymbol{Q}_{\mathrm{s}}$ has been defined as a matrix whose columns are the eigenvectors that constitute the complete signal subspace, including both desired and interfering subspaces.

In conclusion, in a scenario with extremely high power interference, the adaptive algorithm proposed to estimate the generalized eigenvector cor- responding to the maximum generalized eigenvalue converges to the projection of the initial vector $\boldsymbol{w}(0)$ on the signal subspace. Hence, the resultant beamforming weight vector for the second stage of the code reference beamforming depends on the algorithm initialization. However, provided that in this case the first stage is orthogonal to the interference space [7], it turns out that the complete code reference beamforming yields the optimum beamforming weight vector independently of the initial vector $\boldsymbol{w}(0)$ :

$$
\begin{aligned}
\boldsymbol{w}_{\mathrm{CRB}}(i) & =\boldsymbol{R}_{n}^{-1} \boldsymbol{Q}_{\mathrm{s}} \boldsymbol{Q}_{\mathrm{s}}^{\mathrm{H}} \boldsymbol{w}(0)=\boldsymbol{R}_{n}^{-1} \boldsymbol{w}_{\mathrm{opt}} \boldsymbol{w}_{\mathrm{opt}}^{\mathrm{H}} \boldsymbol{w}(0) \\
& =k \boldsymbol{R}_{n}^{-1} \boldsymbol{s}_{\mathrm{d}} .
\end{aligned}
$$

Consequently, the convergence time required to obtain this optimum solution might be considered proportional to the eigenvalue spread of the matrix $\boldsymbol{M}$, as result of considering the minimum eigenvalue of $\boldsymbol{R}_{n}^{-1}$ equal to the inverse of the maximum noise eigenvalue. This spread results equal to the unity for the case of non-directional white noise. In conclusion, the convergence rate of the gradient algorithm is considerably high even in the most disfavorable case, owing to the first stage preprocessing.

\section{References}

[1] L. Acar, R.T. Compton, The performance of an LMS adaptive array with frequency hopped signals, IEEE Trans. Aerospace Electron. Systems AES-21 (3) (May 1985) 360-371.

[2] K. Bakhru, D.J. Torrieri, The maximum algorithm for adaptive arrays and frequency-hopping communications, IEEE Trans. Antennas Propagation AP-32 (9) (September 1984) 919-928.

[3] Y. Bresler, V.U. Reddy, T. Kailath, Optimum beamforming for coherent signal and interferences, IEEE Trans. Acoustics, Speech Signal Process. 36 (6) (June 1988) 833-843.

[4] F. Eken, Use of antenna nulling with frequency-hopping against the follower jammer, IEEE Trans. Antennas Propagation AP-39 (9) (September 1991) 1391-1397.

[5] ETSI-GSM Technical Specification, GSM Radio Aspects, Base Station System, Equipment Specification, GSM 11.21, Version 4.1.0, October 1994.

[6] X. Mestre, M. Nájar, M.A. Lagunas, Two-stage code reference beamformer in mobile communications, Proceedings of the IEEE International Conference on Acoustic, Speech and Signal Processing ICASSP-98, Seattle, May 12-15, 1998, pp. 3309-3312. 
[7] M. Nájar, Conformador de haz de referencia por código de dos etapas: aplicación a la recepción de señales con modulación de saltos frecuenciales, Ph.D. dissertation, October 1996.

[8] M.D. Ortigueira, M.A. Lagunas, Eigendecomposition versus singular value decomposition in adaptive array signal processing, Signal Processing 25 (1991) 35-49.

[9] J.G. Proakis, Digital Communications, McGraw-Hill, New York, 1989.

[10] A. Souloumiac, Blind source detection and separation using second-order non-stationarity, Proceedings of the
IEEE International Conference on Acoustic, Speech and Signal Processing ICASSP-95, Detroit, May 8-12, 1995, pp. 1912-1915.

[11] B. Suard, A.F. Naguib, G. Xu, A. Paulraj, Performance of CDMA mobile communication systems using antenna arrays, Proceedings of the IEEE International Conference on Acoustic, Speech and Signal Processing ICASSP-93, Minneapolis, April 27-30, 1993, pp. IV153-IV156.

[12] M. Viberg, Sensor array processing using gated signals, IEEE Trans. Acoustic, Speech Signal Process. ASSP-37 (3) (March 1989) 447-450. 